\title{
Increased Serum Pigment Epithelium-Derived Factor in Women with Gestational Diabetes Is Associated with Type 2 Diabetes
}

\author{
Tong-Huan Li, Chun-Jian Qiu, Xiao-Juan Yu, Dan-Dan Liu, Peng-Fei Zhou, and Liang Wu \\ Department of Endocrinology, No. 81 Hospital of PLA, Nanjing 210002, China \\ Correspondence should be addressed to Tong-Huan Li; tonghl19@163.com
}

Received 4 January 2015; Revised 11 March 2015; Accepted 12 March 2015

Academic Editor: Kristin Eckardt

Copyright (C) 2015 Tong-Huan Li et al. This is an open access article distributed under the Creative Commons Attribution License, which permits unrestricted use, distribution, and reproduction in any medium, provided the original work is properly cited.

\begin{abstract}
Background. Pigment epithelium-derived factor (PEDF) is demonstrated to be elevated in diabetes patients. However, no reports have emerged in pregnant women with gestational diabetes mellitus (GDM). This study was undertaken to investigate serum PEDF levels in GDM women and to evaluate PEDF as a biomarker to predict diabetes postpartum. Methods. Serum PEDF concentration and clinical characteristics were detected in the pregnant women with GDM ( $n=120)$ and without GDM (control group, $n=120)$. Results. PEDF levels were elevated in subjects with GDM versus controls. Univariate correlations showed that serum PEDF levels were positively correlated with fasting glucose and fasting insulin levels, respectively, and negatively correlated with adiponectin. Receiver operating characteristic (ROC) analysis demonstrated that the AUC of serum PEDF for diabetes mellitus in women postpartum was 0.893 . Conclusion. Serum PEDF was elevated in pregnant women with GDM, which is probably an early detection marker for predicting development of GDM to diabetes mellitus.
\end{abstract}

\section{Introduction}

Gestational diabetes mellitus (GDM) is any degree of glucose intolerance with onset or first recognition during pregnancy, and the prevalence of GDM is rapidly increasing [1]. Most women with GDM revert to normal glucose metabolism after delivery of their babies; however, they are at risk of developing type 2 diabetes later in life as are their offspring. It has been reported that prevalence of the metabolic syndrome and type 2 diabetes in women with previous GDM is higher than in the general ones $[2,3]$. The offspring of the women with GDM also have increased risk of metabolic syndrome or diabetes [4]. Therefore, GDM is an important public health concern, which threatens the health of pregnant women and their offspring.

Pigment epithelium-derived factor (PEDF) is first considered to be a natural extracellular component of the retina and its decreased level has been shown to participate in the pathogenesis of diabetic retinopathy $[5,6]$. The production of circulating PEDF is mainly considered to be liver and adipose tissue [7]. In recent years, PEDF was reported to have potent anti-inflammatory, antiangiogenic, antioxidant, and microvascular protective properties [8-10]. Researchers also found that PEDF is associated with insulin sensitivity, diabetes mellitus, and its complications [11-13]. Clinical and epidemiological studies have proven that serum PEDF is increased in type 2 diabetes mellitus (T2DM) patients [14]. However, if it is good marker to predict the GDM development to diabetes after delivery is still unknown. In present study, the serum PEDF was detected at gestation and the predictive potential was evaluated in 240 pregnant women.

\section{Methods and Materials}

2.1. Subjects. The ethics committee of No. 81 Hospital of PLA approved this study, and all participants gave informed consent. 240 pregnant Chinese women were recruited at the time of antepartum screening for GDM in this study and a retrospective analysis was performed.

2.2. Definitions of GDM and Type 2 Diabetes. The diagnosis of GDM was performed as recommended by the American Diabetes Association [15]. Briefly, a $50 \mathrm{~g}$ OGCT was performed without prior fasting at 24-28 weeks of gestation. Women with a measured $1 \mathrm{~h}$ plasma glucose level above $7.8 \mathrm{mmol} / \mathrm{L}$ 
TABLE 1: Clinical and biochemical characteristics of gestational diabetes mellitus (GDM) patients and controls.

\begin{tabular}{|c|c|c|c|}
\hline Parameter & $\operatorname{GDM}(n=120)$ & Control $(n=120)$ & $P$ value \\
\hline Age (years) & $30.14 \pm 3.35$ & $29.87 \pm 3.07$ & 0.421 \\
\hline Week of gestation & $27.4 \pm 1.5$ & $26.8 \pm 1.2$ & 0.581 \\
\hline Weight (kg) & $56.71 \pm 5.48$ & $55.98 \pm 5.21$ & 0.204 \\
\hline Height $(\mathrm{cm})$ & $158.42 \pm 4.51$ & $158.01 \pm 4.27$ & 0.618 \\
\hline Prepregnancy BMI $\left(\mathrm{kg} / \mathrm{m}^{2}\right)$ & $23.49 \pm 3.47$ & $22.31 \pm 2.71$ & 0.178 \\
\hline Total cholesterol (mg/dl) & $175.01 \pm 21.31$ & $183.14 \pm 23.14$ & 0.214 \\
\hline Triglyceride (mg/dl) & $91.23 \pm 51.31$ & $84.12 \pm 43.56$ & 0.276 \\
\hline HDL-C (mg/dl) & $47.19 \pm 10.3$ & $68.12 \pm 9.6$ & 0.105 \\
\hline LDL-C (mg/dl) & $107.13 \pm 20.18$ & $97.18 \pm 19.25$ & 0.205 \\
\hline Glucose $0 \mathrm{~h}(\mathrm{mmol} / \mathrm{L})$ & $4.92 \pm 0.91$ & $4.21 \pm 0.42$ & 0.032 \\
\hline Glucose $1 \mathrm{~h}(\mathrm{mmol} / \mathrm{L})$ & $10.7 \pm 1.72$ & $7.12 \pm 1.32$ & $<0.01$ \\
\hline Glucose $2 \mathrm{~h}(\mathrm{mmol} / \mathrm{L})$ & $9.02 \pm 1.03$ & $6.14 \pm 1.26$ & $<0.01$ \\
\hline Fasting insulin (pmol/l) & $68.13 \pm 12.12$ & $55.35 \pm 7.12$ & 0.037 \\
\hline HOMA-IR & $2.67 \pm 1.23$ & $1.62 \pm 0.67$ & $<0.01$ \\
\hline Adiponectin (mg/l) & $12.34 \pm 6.12$ & $23.05 \pm 7.12$ & $<0.01$ \\
\hline $\operatorname{PEDF}(\mu \mathrm{g} / \mathrm{ml})$ & $5.02 \pm 2.12$ & $3.34 \pm 1.23$ & $<0.01$ \\
\hline
\end{tabular}

The difference of values between GDM patients and controls was performed by independent-samples $t$-test; $P<0.05$ was considered to be significantly different; BMI: body mass index; HDL-C: high-density lipoprotein cholesterol; LDL-C: low-density lipoprotein cholesterol; HOMA-IR: homeostasis model assessment of insulin resistance; PEDF: pigment epithelium-derived factor.

were considered to have a positive screening test and underwent a diagnostic 100-g OGTT. For the diagnostic 100-g OGTT, fasting plasma glucose levels were initially measured, followed by three plasma glucose measurements taken at hourly intervals (1, 2, and $3 \mathrm{~h}$ ) after ingestion of a glucose load. Women with two or more plasma glucose levels as followings are defined to be GDM: fasting glucose of $\geqslant 5.3 \mathrm{mmol} / \mathrm{L}, 1 \mathrm{~h}$ glucose of $\geqslant 10.0 \mathrm{mmol} / \mathrm{L}, 2 \mathrm{~h}$ glucose of $\geqslant 8.6 \mathrm{mmol} / \mathrm{L}$, and $3 \mathrm{~h}$ glucose of $\geqslant 7.8 \mathrm{mmol} / \mathrm{L}$. At 3 months postpartum, OGTT was also performed in all subjects to diagnose T2DM. Criteria for the diagnosis of diabetes are as follows. (1) A1C $\geqslant 6.5 \%$ : the test should be performed in a laboratory using a method that is NGSP certified and standardized to the DCCT assay. (2) $\mathrm{FPG} \geqslant 7.0 \mathrm{mmol} / \mathrm{L}$ is defined as no caloric intake for at least $8 \mathrm{~h}$. (3) $2 \mathrm{~h}$ serum glucose $\geqslant 11.1 \mathrm{mmol} / \mathrm{L}$ during an OGTT: the test should be performed as described by the World Health Organization, using a glucose load containing the equivalent of $75 \mathrm{~g}$ anhydrous glucose dissolved in water. (4) In a patient with classic symptoms of hyperglycemia or hyperglycemic crisis, a random plasma glucose $\geqslant 11.1 \mathrm{mmol} / \mathrm{L}$. In the absence of unequivocal hyperglycemia, criteria (1)-(3) should be confirmed by repeat testing.

2.3. Physical Body Assessment. Body weight and height were measured using an electronic weighing scale and stadiometer (HGM-200, Hangzhou, China). BMI was calculated as follows: body weight $(\mathrm{kg}) /[$ height $(\mathrm{m}) \times$ height $(\mathrm{m})]$.

2.4. Biochemical Analysis. All blood samples were obtained in the morning after the patients had fasted overnight at 24-32 weeks of gestation. Serum glucose, total cholesterol, triglycerides, HDL-c, LDL-c, and fasting insulin concentrations were determined with standard laboratory methods using an automatic biochemical analyzer (HF400; Shanghai, China). HOMA-IR was calculated as follows: (fasting serum glucose $\times$ fasting insulin)/22.5. serum adiponectin and PEDF concentrations were measured by ELISA kits (R\&D Systems, USA) according to the operation instruction. The sensitivity in this assay was up to $18.75 \mathrm{pg} / \mathrm{mL}$. The specificity for detection of PEDF was excellent, and no significant cross-reactivity or interference between PEDF and analogues was observed.

2.5. Statistical Analysis. Data were analyzed using the Statistical Package for the Social Sciences (SPSS, version 17.0; USA). The skewed variables were logarithmically transformed for statistical analysis. All data were expressed as means \pm SD. Method of independent-samples $t$-test was used to compare means between GDM group and control group. A general linear model analysis was also performed and Pearson's correlation coefficients were used to examine relationships between PEDF and clinical characteristics. A receiver operating characteristic (ROC) curve was drawn to assess diagnostic accuracy; the number of GDM patients included to the ROCAUC analysis was 110 (10 subjects were lost to followup). $P<0.05$ was considered statistically significant.

\section{Results}

3.1. Subjects Characteristics. The subject characteristics are shown in Table 1. Age, week of gestation, prepregnancy $\mathrm{BMI}$, total cholesterol, triglyceride, HDL-C, and LDL-C in GDM women were not significantly different compared with control $(P>0.05)$. The GDM women had higher serum levels of $0 \mathrm{~h}, 1 \mathrm{~h}$, and $2 \mathrm{~h}$ glucose values during $75 \mathrm{~g}$ OGTT, fasting insulin, and HOMA-IR than that in control ones $(P<0.05)$. In addition, serum adiponectin level was markedly reduced 
TABLE 2: Univariate correlations with serum pigment epitheliumderived factor (PEDF) levels in gestational diabetes mellitus (GDM) patients.

\begin{tabular}{lcc}
\hline Parameter & $r$ & $P$ value \\
\hline Age & 0.05 & 0.721 \\
Week of gestation & 0.02 & 0.621 \\
Weight & 0.08 & 0.435 \\
Height & 0.01 & 0.918 \\
Prepregnancy BMI & 0.17 & 0.08 \\
Total cholesterol & -0.07 & 0.312 \\
Triglyceride & -0.1 & 0.255 \\
HDL-C & 0.07 & 0.413 \\
LDL-C & -0.04 & 0.578 \\
Glucose 0 h & 0.2 & 0.031 \\
Glucose 1h & 0.37 & $<0.01$ \\
Glucose 2 h & 0.34 & $<0.01$ \\
Fasting insulin & 0.24 & 0.011 \\
HOMA-IR & 0.371 & $<0.01$ \\
Adiponectin & -0.412 & $<0.01$ \\
\hline
\end{tabular}

Univariate correlations were performed by a general linear model analysis and Pearson's correlation coefficients $(r)$ were used to examine relationships between PEDF and clinical characteristics; $n=120 ; P<0.05$ was considered to be statistically related; BMI: body mass index; HDL-C: high-density lipoprotein cholesterol; LDL-C: low-density lipoprotein cholesterol; HOMAIR: homeostasis model assessment of insulin resistance.

and PEDF concentration was markedly elevated in GDM women versus control subjects.

3.2. Univariate Correlations. As shown in Table 2, serum PEDF level was positively correlated with fasting glucose, $1 \mathrm{~h}$ and $2 \mathrm{~h}$ glucose during $75 \mathrm{~g}$ OGTT $(P<0.05)$. Serum adiponectin value was negatively correlated with PEDF level $(P<$ $0.05)$. However, PEDF concentration did not show an association with age, week of gestation, prepregnancy BMI, and blood lipids levels $(P>0.05)$. As shown in Table 3 , glucose $1 \mathrm{~h}$, glucose $2 \mathrm{~h}$, and HOMA-IR remained in the multivariate linear regression analysis as independent predictors with $P<$ 0.01 .

3.3. ROC Curve Analysis. 3 months postpartum, OGTT was performed in all subjects and 20 GDM women were diagnosed to be T2DM. The diagnostic value of serum PEDF (detected in pregnant weomen) was evaluated by ROC analysis (Figure 1). The values of AUC, optimal cut-off value, sensitivity, and specificity for PEDF, respectively, were $0.893(95 \%$ confidence interval: $0.853-0.933 ; P<0.01), 4.23 \mu \mathrm{g} / \mathrm{mL}$, 0.900 , and 0.667 .

\section{Discussion}

Pigment epithelium-derived factor (PEDF) was first defined in conditioned medium of human retinal pigment epithelial cells as a factor with neuronal differentiating activity $[5,16]$. In recent years, researchers found that PEDF is associated with diabetes mellitus and its complications [17].
TABLE 3: Multivariate linear regression analysis of serum pigment epithelium-derived factor (PEDF) levels in gestational diabetes mellitus (GDM) patients.

\begin{tabular}{lcc}
\hline Parameter & Beta & $P$ value \\
\hline Glucose $1 \mathrm{~h}$ & 0.31 & $<0.01$ \\
Glucose $2 \mathrm{~h}$ & 0.29 & $<0.01$ \\
HOMA-IR & 0.332 & $<0.01$ \\
\hline
\end{tabular}

$n=120 ; P<0.05$ was considered statistically significant; HOMA-IR: homeostasis model assessment of insulin resistance.

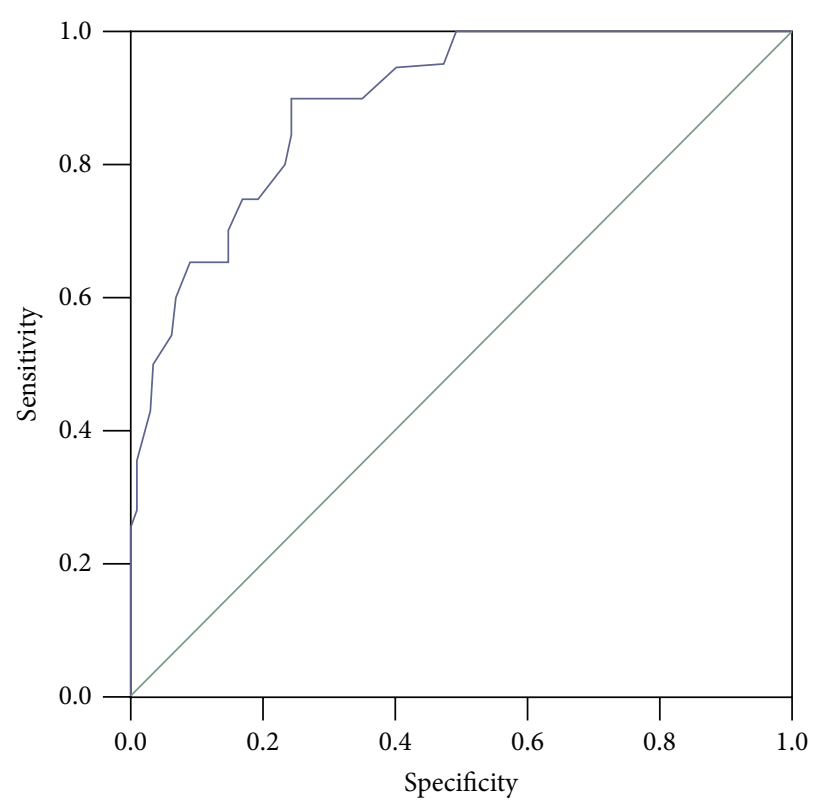

FIGURE 1: ROC curve showing the predictive probabilities of serum PEDF for type 2 diabetes mellitus. The number of GDM patients included to the ROC analysis was 110 (10 subjects were lost to followup).

Gestational diabetes mellitus (GDM) has an increasing prevalence during the last decade [18]. The precise mechanisms underlying GDM remain unclear, and gestational age, progestation $\mathrm{BMI}$, and family history are included in the risk factors in development of GDM. GDM is once thought to be a simply impaired glucose tolerance temporarily associated with pregnancy [19]. However, it is a medical condition associated with long-term consequences for both maternal and fetal morbidity, including the delivery trauma, increase of diabetes mellitus, and obesity incidence $[20,21]$. Therefore, prevention and reasonable control of GDM are important for the health of baby and mother.

There are emerging evidences to prove that PEDF is involved in microvascular complications, hyperglycemia, inflammation, and cardiovascular diseases [22]. Yamagishi et al. found that serum PEDF is elevated in proportion to the accumulation of the number of components of the metabolic syndrome [23]. PEDF has been demonstrated to inhibit the advanced glycation end product in adipocytes but causes insulin resistance in skeletal smooth muscle cells $[7,24]$. In in vivo experiments, it is shown that PEDF could improve 
metabolic derangements by suppressing the inflammatory and oxidative reactions in adipose tissues of rats [25]. The scholars considered increased PEDF as a counter-system against obesity-related metabolic derangements. In present study, PEDF was measured at 24-32 weeks of gestation in all 240 pregnant women with or without GDM. The results showed that serum PEDF was elevated in GDM women compared to that in controls, and univariate correlations data in pregnant women demonstrated that serum PEDF level was positively related with fasting glucose and HOMA-IR, a marker of insulin resistance in high-risk patients for cardiovascular disease [26], which implied that GDM women had an abnormal serum PEDF. Increased PEDF was also observed in diabetes patients in previous studies [11, 14]. To our knowledge, this is the first study in pregnant women with GDM detecting serum PEDF level. Serum increased PEDF levels probably contributed to be a counter-system against metabolic abnormal in GDM women.

To further investigate the influences of serum PEDF during pregnancy on postpartum glucose levels, all subjects were followed up postpartum. At 3 months postpartum, 20 of 120 women previously with GDM were diagnosed to T2DM. The ROC curve analysis showed that the AUC of PEDF for T2DM of GDM women was 0.893 , and the sensitivity and specificity for PEDF were 0.900 and 0.667 , respectively, which implied that PEDF is probably an early marker for predicting T2DM after delivery in women previously with GDM. As described above, gestational age, progestation BMI, and family history of diabetes mellitus are important risk factors for GDM. In our study, the subjects in GDM group and control had no significant difference in gestational age and BMI, and all participants and their parents have no history of diabetes mellitus. We hold that it is necessary to take actions to prevent the development of T2DM in gestation if the serum PEDF concentration is abnormal. However, a larger sample size in different areas should be investigated to confirm this conclusion.

In summary, our study showed that serum PEDF was elevated in pregnant women with GDM, which is probably an early detection marker for predicting development of GDM to T2DM. Further studies are needed to explore the clinical prediction of PEDF detection in pregnant women.

\section{Conflict of Interests}

All authors declare that they have no conflict of interests.

\section{References}

[1] M. M. Gabir, R. L. Hanson, D. Dabelea et al., "The 1997 American Diabetes Association and 1999 World Health Organization criteria for hyperglycemia in the diagnosis and prediction of diabetes," Diabetes Care, vol. 23, no. 8, pp. 1108-1112, 2000.

[2] J. Lauenborg, E. Mathiesen, T. Hansen et al., "The prevalence of the metabolic syndrome in a Danish population of women with previous gestational diabetes mellitus is three-fold higher than in the general population," The Journal of Clinical Endocrinology \& Metabolism, vol. 90, no. 7, pp. 4004-4010, 2005.

[3] L. Bellamy, J.-P. Casas, A. D. Hingorani, and D. Williams, “Type 2 diabetes mellitus after gestational diabetes: a systematic review and meta-analysis," The Lancet, vol. 373, no. 9677, pp. 1773-1779, 2009.

[4] P. Damm, "Future risk of diabetes in mother and child after gestational diabetes mellitus," International Journal of Gynecology and Obstetrics, vol. 104, supplement 1, pp. S25-S26, 2009.

[5] J. Tombran-Tink, G. G. Chader, and L. V. Johnson, "PEDF: a pigment epithelium-derived factor with potent neuronal differentiative activity," Experimental Eye Research, vol. 53, no. 3, pp. 411-414, 1991.

[6] S. Yamagishi, T. Matsui, and H. Inoue, "Inhibition by advanced glycation end products (AGEs) of pigment epithelium-derived factor (PEDF) gene expression in microvascular endothelial cells," Drugs under Experimental and Clinical Research, vol. 31, no. 5-6, pp. 227-232, 2005.

[7] S. Famulla, D. Lamers, S. Hartwig et al., "Pigment epitheliumderived factor (PEDF) is one of the most abundant proteins secreted by human adipocytes and induces insulin resistance and inflammatory signaling in muscle and fat cells," International Journal of Obesity, vol. 35, no. 6, pp. 762-772, 2011.

[8] S. X. Zhang, J. J. Wang, G. Gao, K. Parke, and J.-X. Ma, "Pigment epithelium-derived factor downregulates vascular endothelial growth factor (VEGF) expression and inhibits VEGF-VEGF receptor 2 binding in diabetic retinopathy," Journal of Molecular Endocrinology, vol. 37, no. 1, pp. 1-12, 2006.

[9] Y. Liu, L. F. Leo, C. McGregor, A. Grivitishvili, C. J. Barnstable, and J. Tombran-Tink, "Pigment epithelium-derived factor (PEDF) peptide eye drops reduce inflammation, cell death and vascular leakage in diabetic retinopathy in Ins $2^{\text {Akita }}$ mice," Molecular Medicine, vol. 18, pp. 1387-1401, 2012.

[10] M. Elahy, S. Baindur-Hudson, V. F. Cruzat, P. Newsholme, and C. R. Dass, "Mechanisms of PEDF-mediated protection against reactive oxygen species damage in diabetic retinopathy and neuropathy," Journal of Endocrinology, vol. 222, no. 3, pp. R129R139, 2014.

[11] M. Sabater, J. M. Moreno-Navarrete, F. J. Ortega et al., "Circulating pigment epithelium-derived factor levels are associated with insulin resistance and decrease after weight loss," Journal of Clinical Endocrinology and Metabolism, vol. 95, no. 10, pp. 47204728, 2010.

[12] K. M. Choi, S. Y. Hwang, H. C. Hong et al., "Clq/TNF-related protein-3 (CTRP-3) and pigment epithelium-derived factor (PEDF) concentrations in patients with type 2 diabetes and metabolic syndrome," Diabetes, vol. 61, no. 11, pp. 2932-2936, 2012.

[13] H. Chen, Z. Zheng, R. Li et al., "Urinary pigment epitheliumderived factor as a marker of diabetic nephropathy," The American Journal of Nephrology, vol. 32, no. 1, pp. 47-56, 2010.

[14] A. Jenkins, S. X. Zhang, A. Gosmanova et al., "Increased serum pigment epithelium derived factor levels in Type 2 diabetes patients," Diabetes Research and Clinical Practice, vol. 82, no. 1, pp. e5-e7, 2008.

[15] American Diabetes Association, "Diagnosis and classification of diabetes mellitus," Diabetes Care, vol. 33, supplement 1, pp. S62-S69, 2010

[16] J. Tombran-Tink and L. V. Johnson, "Neuronal differentiation of retinoblastoma cells induced by medium conditioned by human RPE cells," Investigative Ophthalmology and Visual Science, vol. 30, no. 8, pp. 1700-1707, 1989.

[17] S.-I. Yamagishi, T. Matsui, K. Nakamura, S. Ueda, Y. Noda, and T. Imaizumi, "Pigment epithelium-derived factor (PEDF): its potential therapeutic implication in diabetic vascular complications," Current Drug Targets, vol. 9, no. 11, pp. 1025-1029, 2008. 
[18] D. Dabelea, J. K. Snell-Bergeon, C. L. Hartsfield, K. J. Bischoff, R. F. Hamman, and R. S. McDuffie, "Increasing prevalence of gestational diabetes mellitus (GDM) over time and by birth cohort: Kaiser Permanente of Colorado GDM Screening Program," Diabetes Care, vol. 28, no. 3, pp. 579-584, 2005.

[19] American Diabetes Association, "Diagnosis and classification of diabetes mellitus," Diabetes Care, vol. 29, supplement 1, pp. S43-S48, 2006.

[20] C. Capula, E. Chiefari, A. Vero, D. P. Foti, A. Brunetti, and R. Vero, "Prevalence and predictors of postpartum glucose intolerance in Italian women with gestational diabetes mellitus," Diabetes Research and Clinical Practice, vol. 105, no. 2, pp. 223230, 2014.

[21] D. Wang, W. Zhu, J. Li, C. An, and Z. Wang, "Serum concentrations of fibroblast growth factors 19 and 21 in women with gestational diabetes mellitus: association with insulin resistance, adiponectin, and polycystic ovary syndrome history," PLoS ONE, vol. 8, no. 11, Article ID e81190, 2013.

[22] A. J. Jenkins, S. X. Zhang, K. G. Rowley et al., "Increased serum pigment epithelium-derived factor is associated with microvascular complications, vascular stiffness and inflammation in Type 1 diabetes," Diabetic Medicine, vol. 24, no. 12, pp. 1345-1351, 2007.

[23] S.-I. Yamagishi, T. Matsui, K. Nakamura, M. Takeuchi, and T. Imaizumi, "Pigment epithelium-derived factor (PEDF) prevents diabetes- or advanced glycation end products (AGE)elicited retinal leukostasis," Microvascular Research, vol. 72, no. 1-2, pp. 86-90, 2006.

[24] T. Yoshida, S. Yamagishi, K. Nakamura et al., "Pigment epithelium-derived factor (PEDF) ameliorates advanced glycation end product (AGE)-induced hepatic insulin resistance in vitro by suppressing Rac-1 activation," Hormone and Metabolic Research, vol. 40, no. 9, pp. 620-625, 2008.

[25] T. Matsui, Y. Nishino, A. Ojima, S. Maeda, N. Tahara, and S.-I. Yamagishi, "Pigment epithelium-derived factor improves metabolic derangements and ameliorates dysregulation of adipocytokines in obese type 2 diabetic rats," The American Journal of Pathology, vol. 184, no. 4, pp. 1094-1103, 2014.

[26] N. Tahara, S.-I. Yamagishi, A. Tahara, M. Takeuchi, and T. Imaizumi, "Serum levels of pigment epithelium-derived factor, a novel marker of insulin resistance, are independently associated with fasting apolipoprotein B48 levels in humans," Clinical Biochemistry, vol. 45, no. 16-17, pp. 1404-1408, 2012. 


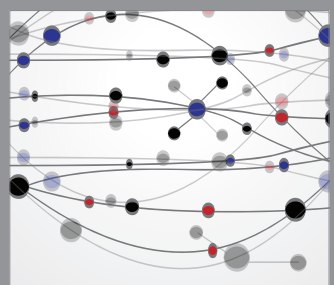

The Scientific World Journal
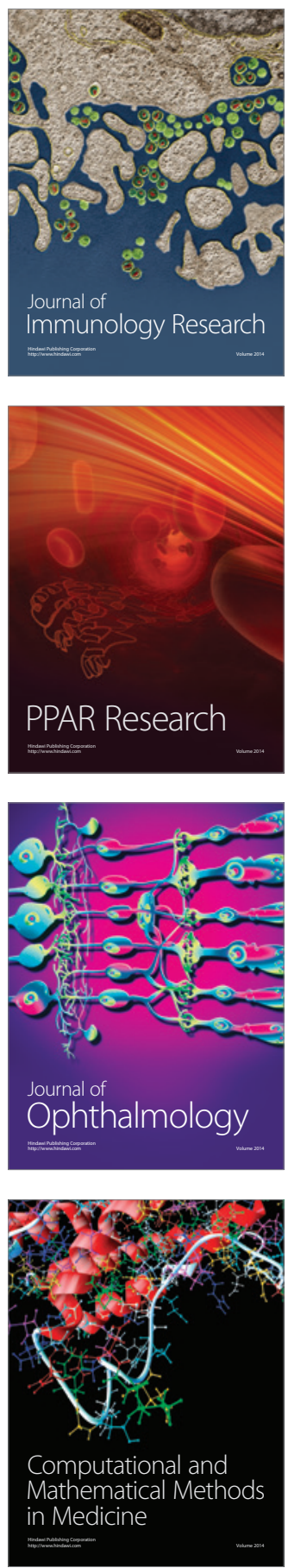

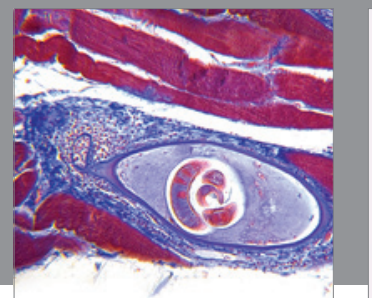

Gastroenterology

Research and Practice
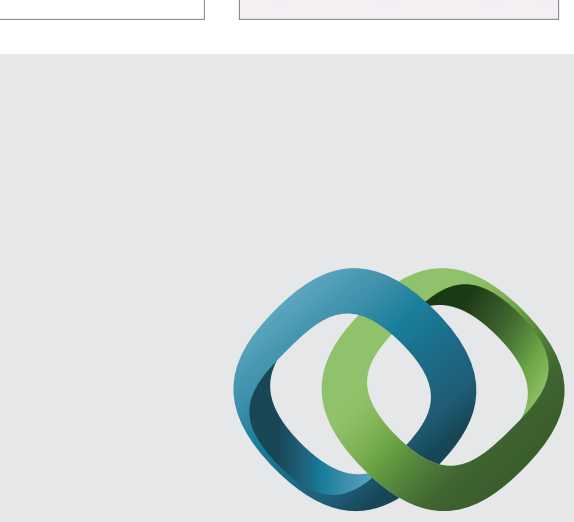

\section{Hindawi}

Submit your manuscripts at

http://www.hindawi.com
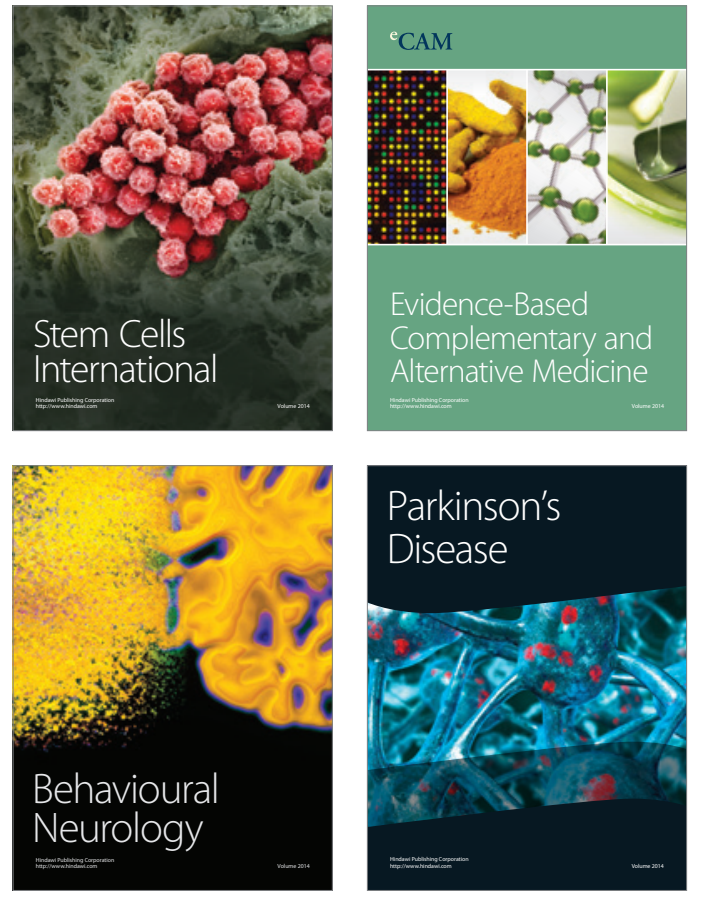
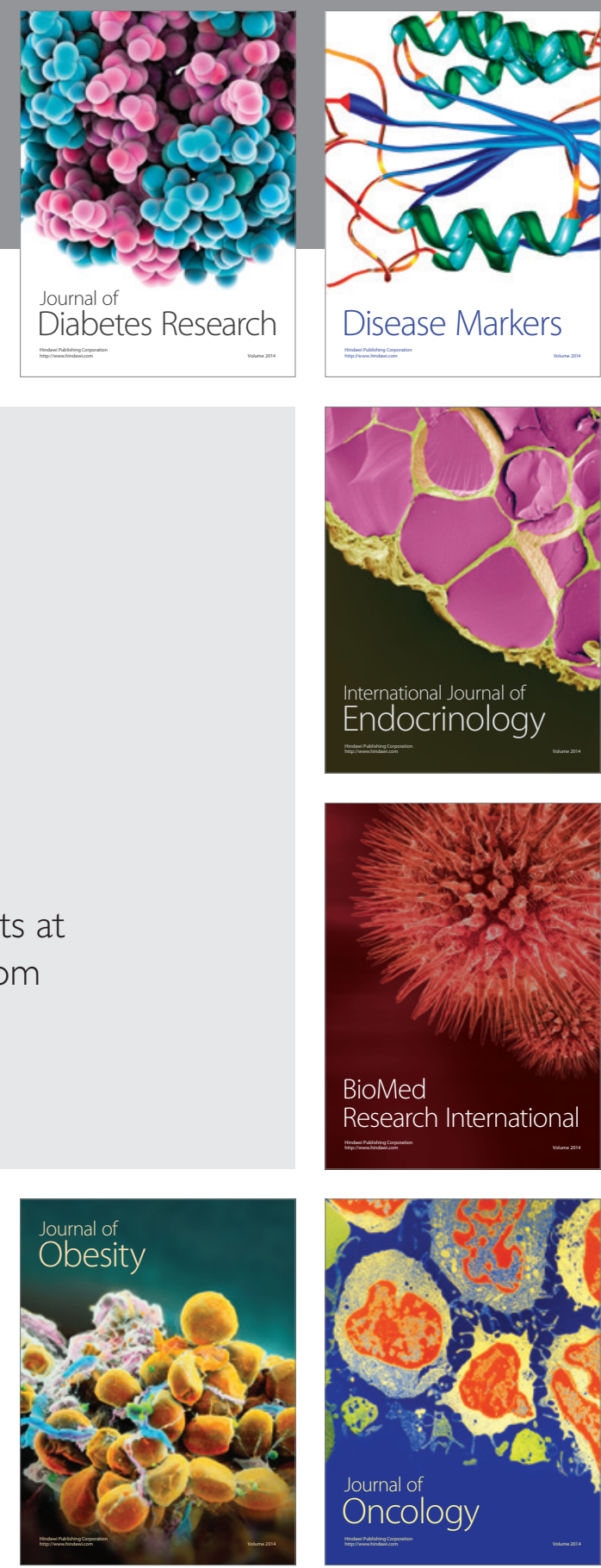

Disease Markers
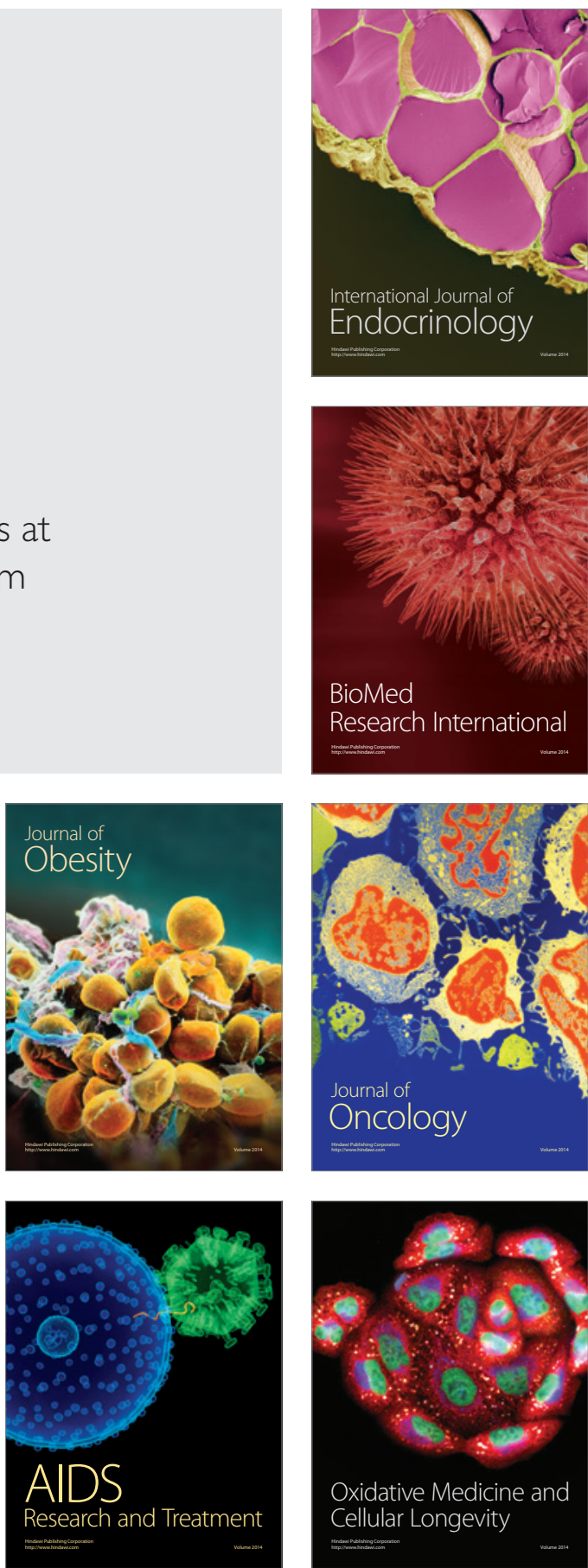